anorexics might show clinical improvement following mirror confrontation.

In contrast, preferred treatment for dysmorphophobia might involve the reverse behavioural approach. Support for this hypothesis comes from clinical accounts of patients who report themselves much improved after cosmetic surgery when objective ratings of their appearance indicate no significant change.

The patients who do well have a supportive confdant, an optimistic attitude, and have been prevented from looking at the particular body part, which is shrouded in dressings after surgery. This arguably may allow the dissolution of the distorted body image, which in part may be a manifestation of heightened cognitive awareness for, and the preferential visual scanning of, the body part in question. A similar cognitive-behavioural approach could be envisaged for over-focused dysmorphophobic patients.

University of Western Ontario

LAURENCE JEROME

London, Ontario

Canada N6G 2 K3

References

HAY, G. G. (1970) Dysmorphophobia. British Journal of Psychiatry, $116,399-406$.

Jerome, L. (1980) A Study of the Perception of Body Image in Patients Requesting Cosmetic Rhinoplasty. MSc thesis, University of Manchester.

NORRIS, D. L. (1984) The effects of mirror confrontation on selfestimation of body dimensions in anorexia nervosa, bulimia, and two control groups. Psychological Medicine, 14, 835-842.

\section{Organic Factors in Catatonia}

SIR: Though Wilcox \& Nasrallah (Journal, December 1986, 149, 782-784) note that catatonia has been reported in non-schizophrenic individuals, they fail to mention that in a study of 55 consecutively admitted catatonic patients Abrams \& Taylor (1976) found a preponderance of cases with manic (34) and depressive (5) disorders but only four with schizophrenia. Similarly, of the 25 cases studied by Barnes et al (1986), ten were idiopathic and nine had a depressive illness. There was a single case of schizophrenia. Both the idiopathic and the affective groups had a high incidence of recurrent episodes and a family history of catatonia. These findings confirm Kahlbaum's original description of catatonia as a non-specific syndrome, rather than a disease entity, with a wide range of causes, of which affective disorders appear to be the most common.

The undue emphasis on the association of catatonia with schizophrenia is also pertinent to the tragic patient reported by Ainsworth (Journal, January $1987,150,110-112)$. In view of the striking affective changes in her patient's mental state (as well as the family history of depression and mutism), an affective disorder, possibly mania, seems a more appropriate diagnosis than catatonic schizophrenia. Though reported as a case of Stauder's lethal catatonia, no evidence is presented of its characteristic features, i.e. mounting fever and extreme hyperactivity progressing to stuporous exhaustion and death. Clinically it resembles the more familiar benign variety of Kahlbaum (Mann et al, 1986).

Ainsworth raises several questions which have an important bearing on the treatment of such patients. Though amylobarbitone sodium interview is reportedly helpful in distinguishing catatonia of psychiatric origin from that with a toxic-metabolic or neurological basis, its value in the lethal variety is uncertain. Whether a putative affective disorder developed subsequent to a sub-clinical viral encephalitis or predisposed her patient to increased infection is a moot point (Wilson, 1976), but the brief return to normality after i.v. diazepam would argue against the usefulness of such techniques rather than render a diagnosis of encephalitis less probable in light of the clinical and pathological findings. Orthostatic hypotension due to the high doses of chlorpromazine could have caused the patient to fall, and while the cause of death seems unrelated to the catatonia, a viral encephalitis may well have compromised her gag reflex, resulting in fatal aspiration. The outcome for patients with benign catatonia, whether of psychiatric or other origins, is described as excellent and most recover spontaneously or rapidly after ECT, although a high rate of recurrence has been reported, particularly among those with a family history of catatonia (Barnes et al, 1986). In patients with lethal catatonia ECT may also be dramatically effective, and perhaps lifesaving (Mann et al, 1986).

Finally, several authors have commented on the similarities between lethal catatonia and the neuroleptic malignant syndrome, which may well be a drug-induced variety of the former. Because of the dangers posed by neuroleptic treatment in iatrogenic and other forms of lethal catatonia, as well as evidence that some patients develop stupor and fever only after the initiation of such treatment, a prudent approach would be to discontinue its use in benign catatonia too. In view of its efficacy, ECT would appear to be the treatment of choice in catatonia, although in some cases of the lethal variety other 
agents, e.g. dopamine agonists such as bromocriptine or dantrolene, may be necessary initially.

Towers Hospital

KAREL De PAUW

Gipsy Lane

Leicester LE5 OTD

Bassetlaw District General Hospital

KRYSTYNA SZULECKA

Worksop

Notts S81 OBD

\section{References}

Abrams, R. \& TAYLOR, M. A. (1976) Catatonia: a prospective clinical study. Archives of General Psychiatry, 33, 579-581.

Barnes, M. P., SAunders, M., Walls, T. J., Saunders, I. \& Kirk, C. A. (1986) The syndrome of Karl Ludwig Kahlbaum. Journal of Neurology, Neurosurgery and Psychiatry, 49, 991-996.

ManN, S. C., Carofr, S. N., Bleier, H. R., Welz, W. K. R., Kling, M. A. \& HAYASHIDA, M. (1986) Lethal catatonia. American Journal of Psychiatry, 143, 1374-1381.

WILSON, L. G. (1976) Viral encephalopathy mimicking functional psychosis. American Journal of Psychiatry, 133, 165-170.

SIR: Wilcox \& Nasrallah (Journal, December 1986, $149,782-784$ ) suggest that the reason why catatonia has become something of a rarity is that brain injury in childhood, which the authors postulate as predisposing to catatonia, has declined in frequency.

I work in the field of mental handicap, in which brain damage is considered, in many instances, to be at the root of the handicap, particularly in profoundly affected patients. I have seen hardly any mentally handicapped individuals suffering from catatonia, although only recently I have been treating someone with this condition. If my experience is shared by other psychiatrists practising mental handicap, this would be an argument against the hypothesis that an organic brain condition is a forerunner of catatonia, unless one were to say that in the mentally handicapped catatonia, for reasons to do with the level of intelligence, is not a feature.

A survey of the incidence of catatonia in the mentally handicapped would throw light on the attractive theory put forward by Wilcox \& Nasrallah.

I. Freedman

Monyhull Hospital

Monyhull Hall Road

Birmingham

$B 303 Q B$

\section{A Case of Resistant Schizophrenia}

SIR: I wish to comment on the treatment of this unfortunate young man (Mr A) (Journal, December $1986,149,789-793)$. The record of therapeutic failure includes a trial of electroconvulsive therapy: "He was treated with six ECT for a possible affective component to his disorder, but with little benefit." Such a course of electroconvulsive therapy was probably inadequate.

Prior to the introduction of neuroleptic drugs, ECT was commonly used in the treatment of patients with schizophrenia. The comparative studies of the 1960 s did not demonstrate a failure of ECT; rather, they demonstrated the equivalence of group results between those patients treated with ECT and those with neuroleptic drugs. The ease of administration, lesser expense, and assumed greater safety of psychotropic drugs, however, led to their replacement of ECT. Recent concerns about tardive dyskinesia, neuroleptic malignant syndrome, and other hazards of neuroleptic drug use (as well as therapeutic failures) have led some clinicians to re-examine the application of ECT in the treatment of schizophrenia.

Friedel (1986) recently reported the successful use of the combination of thiothixene and ECT in eight of nine patients who were non-responders to extended courses of neuroleptic drugs. We have treated nine schizophrenic patients who were neuroleptic and multi-drug treatment failures with the combination of ECT and fluphenazine. Of these, seven have been functioning well in the community for at least one year, and we are encouraged enough to undertake a random assignment trial. However, these patients required an average of $15 \mathrm{ECT}$ in their treatment course, a number greater than is ordinarily given to our depressed patients.

The report by Brandon et al (1985) of the results of schizophrenic patients treated in the Leicestershire study and that of Taylor \& Fleminger (1980) also encourage the use of ECT in schizophrenic patients. These findings were recently endorsed by the NIH Consensus Conference (1985) and by van Valkenberg \& Clayton (1985).

If $\mathrm{Mr} \mathrm{A}$ is still psychotic, a repeat trial of ECT should be considered with a minimum of 12 induced seizures, preferably with bilateral electrode placement, and with minimum durations of $25 \mathrm{~s}$ for each peripheral seizure ( $30 \mathrm{~s}$ central seizure). These treatments should be given while the usual dosages of neuroleptic drug therapy are continued.

\section{Department of Psychiatry \\ State University of New York at Stony Brook USA}

\section{References}

FrIEDEl (1986) The combined use of neuroleptics and ECT in drugresistant schizophrenic patients. Psychopharmacology Bulletin. 22, $928-930$.

Brandon, S., Cowley, P., McDonald, C., Neville, P., Palmer, $R$. 